\title{
A CROSS-SECTIONAL SURVEY ON DOG ECOLOGY AND DOG ANTI-RABIES VACCINATION COVERAGE IN SELECTED AREAS IN SRI LANKA
}

\author{
R.M.S. Pimburage ${ }^{1}$, P.A.L. Harischandra ${ }^{1}$, M. Gunatilake ${ }^{2}$, D.N. Jayasinhe ${ }^{1}$, \\ A. Balasuriya ${ }^{3}$, R.M.S.K. Amunugama ${ }^{4}$ \\ ${ }^{\prime}$ Public Health Veterinary Services, Ministry of Health, Sri Lanka \\ ${ }^{2}$ Department of Physiology, Faculty of Medicine, University of Colombo, Sri Lanka \\ ${ }^{3}$ Faculty of Medicine, General Sir John Kotelawala Defence University, Sri Lanka \\ ${ }^{4}$ Ministry of Health, Sri Lanka
}

\begin{abstract}
SUMMARY: Dogs are the main transmitter of rabies virus for humans in Sri Lanka. Therefore, updated information on the dog rabies vaccination coverage and dog ecology are essential for launching an effective rabies elimination program in the country.

A descriptive cross sectional study was conducted in 120 clusters. Counts recorded at the vaccination centres were considered as the capture sample. In the recapture sample, data collected on humans and dogs through the questionnaire survey administered on households were considered. Number of dogs vaccinated against rabies and the total number of people in the dog-owning households that attended the vaccination centres were 2508 and 8690 respectively. The household survey of the study included 2207 households, which includes 8650 family members. Among these households, 774 houses had dogs which included 3382 family members.

From the 2933, free-roaming owned dogs and free-roaming dogs with undetermined ownership counted through the transect line, 1413 had been marked in the study areas. Out of 838 owned confined and owned freeroaming dogs found during the questionnaire survey administered on 2207 households, 579 were recorded as recaptured.

The estimated human population of the area surveyed was 34,901. The tabulated ratio of the total dog population in total human was 1:6.7 and owned dog population to human ratio was 1:9.6. The estimated owned dog population was 3630 and the total dog population was 5205. The total dog vaccination coverage is $48 \%$ (2508/5205).Owned (confined and free-roaming) dog vaccination coverage achieved was $69 \%$. Vaccination coverage among owned free-roaming dogs was $54 \%$ and significantly low $(P<0.005)$, compared to owned confined dogs which was $77 \%$. Majority of the dogs owned were local breeds $(90.5 \%)$ aged between 1 and 5 years old. Findings revealed that $34 \%$ of the owned dogs were allowed to roam freely during the day.

During annual mass vaccination campaigns, there should be a method to include dogs in all three categories i.e. Owned confined, owned free-roaming and dogs with undetermined ownership status. Considering confinement status practiced, allowing dogs to roam freely should be discouraged. This could potentially be acquired through implementing educational programs and new legislations bound with legal means.
\end{abstract}

\section{INTRODUCTION}

Rabies is a fatal and neglected zoonotic disease that causes nearly 60,000 human deaths worldwide annually (Hampson et al., 2015). During the last century, several countries have demonstrated that controlling rabies through annual mass vaccination campaigns for domestic dogs is the most reliable and effective way for elimination of rabies (Coleman et al.,1996; Touihri etal., 2011).

Dogs are the main transmitter of rabies for humans in Africa and Asia, including Sri Lanka where rabies is endemic. Out of reported rabies cases in animals, over $96 \%$ are among dogs and $95 \%$ of the human rabies deaths are due to rabid dog bites (Nanayakkara et al., 2003; WHO, 2014). Vaccination of dogs has been identified as an important control measure. The World Health Organization (WHO) recommends that at least $70 \%$ of the domestic dog population should be vaccinated during annual mass vaccination campaigns to achieve and maintain herd immunity among animal reservoirs (Malerczyk, 2012; WHO, 2013; Rupprecht et al., 2008). A study conducted in Sri Lanka in 1997 to evaluate the progress of rabies control measures revealed that the vaccination coverage reached by parental mass vaccination campaigns was $57.6 \%$ (Matter et al., 2000). Studies conducted in Abia State of Nigeria and Thangsong district of Thailand have estimated anti-rabies vaccine coverage of household dogs as $47.9 \%$ and $44 \%$ respectively (Awoyomi et al., 2007; Kongkaew et al., 2004). It is mentioned that vaccination coverage against rabies varies according to demography which includes dog: human ratio, confinement status, the size of the total dog population and free-roaming dog population with undetermined ownership status (Knobel et al., 2008; Wild et al, 2005). Further, an understanding dog ecology has been recognised as a key factor in designing an effective rabies control programme in rabies endemic countries (Gbeminiyi et al., 2014; Coleman et al.,1996; Bearn et 
al.,1982; Bogel et al., 1990).

There had been a considerable variation in the dog: human ratio, which commonly lies between 1:6 and 1:10. A survey done in Africa revealed that the dog: human ratio is 1: 21.2 in urban areas, while rural areas have a ratio of 1:7.4 (Robinson et al.,1996; Kaare et al., 2009; Awoyomi et al., 2007; Oboegbulemand et al., 1989 ). In Sri Lanka, there have been only a few studies that have tried to estimate the dog population size in urban or rural areas; a demography and dog ecology study was conducted in Mirigama, Gampaha which revealed a 1:4 dog: human ratio (Matter et al., 2000) and another study carried out in National Institute of Health Sciences field practice area of Kalutara district in 2006 has revealed a 1: 8.5 dog:human ratio (Pimburage et al., 2011). However the dog population information has not been updated for the last 7 years in Sri Lanka.

An evaluation of dog anti-rabies vaccination coverage and other factors influencing the progress of the on-going rabies control program was a prerequisite to make necessary changes for the future planning of the program. Estimation of the total dog population is essential in order to purchase the number of vaccines required for a national anti-rabies campaign and to determine the vaccination coverage. Further, estimated dog: human ratio can be used to calculate the dog population size for administrative divisions and districts, when the human population in a representative area is known. Therefore, we conducted this study with the aim of updating the dog population ecology and vaccination coverage of dogs against rabies. The findings of this study are expected to be a guide for the national rabies control program in Sri Lanka.

\section{MATERIALS \& METHODS}

\section{Study design and sampling}

This was a cross-sectional analytical study conducted in the year 2013,using the capture recapture method. The study was carried out in two provinces (Western and Southern) of Sri Lanka. Two Divisional Secretariats (DS) from each province were selected based on the average human population (http://www.statistics.gov.lk). Figure 1 illustrates the districts, divisional secretariats (DS) and public health inspector (PHI) areas selected for this study.

The total human population and the dimension of the selected areas are also indicated in Figure 1. Selected PHI areas were Welipanna, Raigama from Matugama and Bandaragama DS areas and Bambaranda and Akuressa from Dikwella and Akuressa DS areas. Among the selected PHI areas in Bandaragama and Akuressa are urban areas while selected PHI areas in Matugama and Dikwella are rural areas. During this study the total area covered was $62.1 \mathrm{~km}^{2}$ (Welipanna PHI- $14 \mathrm{~km}^{2}$, Raigama PHI- $17.5 \mathrm{~km}^{2}$, Bambaranda PHI- $17 \mathrm{~km}^{2}$ and Akurassa PHI- $13.6 \mathrm{~km}^{2}$ ).

In each selected DS division, ten temporary vaccination centres in one public health inspector $(\mathrm{PHI})$ area were

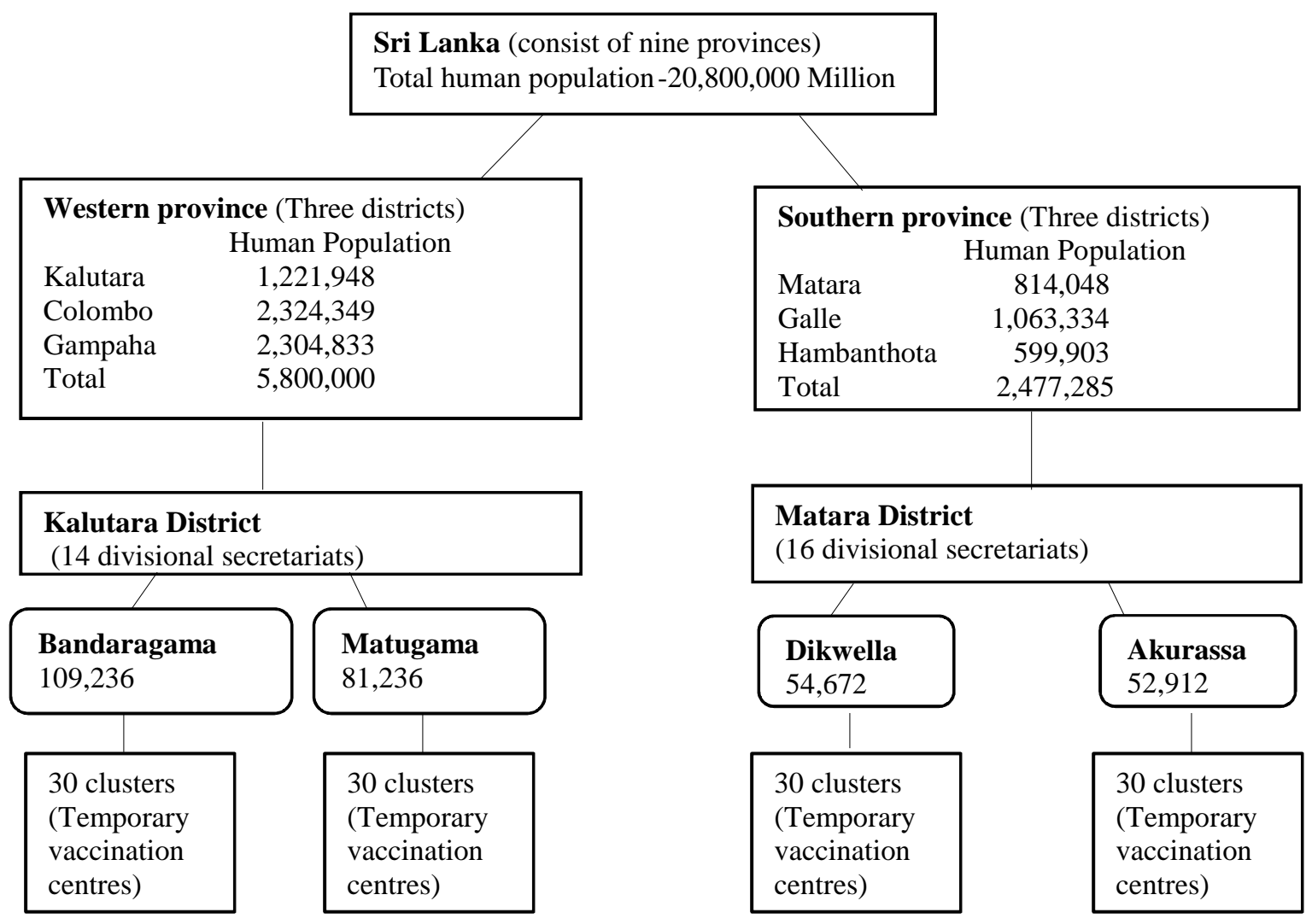

Figure 1: Flow chart indicating sampling 
Dog ecology and dog anti-rabies vaccination

Table 1: Household information on 838 dogs obtained from respondents in 2207 households in study areas

\begin{tabular}{llcc}
\hline Variable & Frequency & Percentage (\%) & ARV coverage \\
\hline $\begin{array}{l}\text { Households with dogs } \\
\text { Sex }\end{array}$ & 0.375 & 37.5 & \\
$\quad$ Male & 662 & 78.9 & $69 \%$ \\
$\quad$ Female & 176 & 21.1 & $66 \%$ \\
Age & & & \\
$\quad 1$ year & 132 & 15.7 & $65 \%$ \\
$\quad 1$ year & 706 & 84.2 & $65 \%$ \\
Breed & & & \\
Local & 759 & 90.5 & $68 \%$ \\
Other & 79 & 9.4 & $78 \%$ \\
Rearing method & & & \\
Caged & 245 & 29.2 & $83 \%$ \\
Leashed & 307 & 36.6 & $71 \%$ \\
Free & 286 & 34.1 & $53 \%$ \\
\hline
\end{tabular}

established for a day for three consecutive days, comprising a total of 30 temporary vaccination centres for the period. Each day capturing and recapturing with transect line counting was carried out.

\section{Duration of the study}

This study was conducted in line with the owned dog (owned confined and owned, free-roaming) vaccination team of national anti-rabies campaign in order to cut down the cost of the study. The national anti-rabies campaign is conducted at the district level and each district has a team of people to cover the district in order to establish an achievable vaccination coverage in the district. Three full days (Area 1: February 19-21, Area 2: April 26-28, Area 3: August 10-12, Area 4: November 15-17) were spent to cover one PHI area (30 clusters $=10$ vaccination centres/per day in 3 days in each PHI area). The area covered by a vaccination centre in one day in the study is approximately $0.8 \mathrm{~km}^{2}$. In-order to cover all the selected PHI areas, 10 months has to be spent as the study followed district antirabies campaigns due to limited funding and human resources.

\section{Categories of dogs included in the study}

The dogs included in the study were considered under following three categories.

- Owned, (continually) confined dogs

- Owned, free-roaming (some or all of the day or night) dogs

- Free-roaming dogs with undetermined ownership status

\section{Capturing phase at the temporary vaccination centre}

As the initial step, one day ahead, people were made aware of the date, time and the place of upcoming antirabies vaccination program using loudspeaker announcements apart from the posters displayed one week prior to the program. Dogs were vaccinated intramuscularly using a commercial anti-rabies vaccine recommended for the National anti-rabies control program. Average distance between two adjoining vaccination centres was $1 \mathrm{~km}$. Five teams of 2 persons operated 10 vaccination centres per day, 5 in the morning and 5 in the evening.

At the point of vaccination, one person was engaged in vaccination and marking of all vaccinated dogs with red collars while the other person collected information about the vaccinated dogs (age, sex, vaccine history) and the households (name and address, number of dogs and number of family members in the household). During the capturing phase free-roaming dogs with undetermined ownership were not vaccinated.

\section{Recapturing through household survey}

Once the vaccination was completed, a household survey was conducted within the $500 \mathrm{~m}$ radius from the vaccination centre. The household survey was done either on the same day afternoon or on the following day morning for vaccination centres conducted in the morning and afternoon respectively to minimize violation of marked-recapture model assumptions i.e. creating a closed population, subjects are not losing their collars, estimating probability of capturing a marked subject is equal to the proportion of marked subjects in the 
population and accurate reporting of all marked subjects. The closest household to the vaccination centre was selected as the first. From there, every other household was visited totalling up to a maximum of 25 . This method was adopted to avoid re-visit of households served by the adjoining vaccination centres. Number of persons in the household and information about their pets [sex, age, breed, confinement status, collar marked or not, whether anti-rabies vaccination was given or not] in the household were recorded. Numbers of marked and unmarked dogs outside the households were also recorded on a separate sheet in parallel with the household survey \{owned dogs, owned free-roaming (dogs who are allowed to roam freely out of the household premises without restrictions) and free-roaming dogs with undetermined ownership\}.

\section{Recapturing through Transect study}

A second recapture of marked and unmarked dogs was carried out in a transect study to estimate the total dog population. Once the vaccination was done, on the following day morning (6.00am - 7.30am), in the area served by the vaccination centres, a daily transect line count of marked and unmarked dogs were done. A person travelling by a car, on a pre-defined transect line following specified roads around and across the temporary vaccination centres, counted (recaptured) all the visible dogs on the roads and inside household premises, with and without collars separately. Inclusion of $500 \mathrm{~m}$ buffer zones during counting prevented counting of dogs that roamed into the study area from adjacent areas.

Using the same method, an overall recount of marked and unmarked dogs was done at the end of three day vaccination program in the entire area included for the program.

\section{Data collection, analysis and ethics}

The Veterinary surgeons and public health inspectors attached to the National anti-rabies control program were trained to collect the data. The interviewer administered questionnaires and data collection sheets used for the survey were pre-tested.

The data were analysed using Statistical Package for Social Sciences (version 10). Descriptive statistics was used to describe the information. The associations between dog confinement status and rabies vaccination coverage were assessed using chi-square test and comparison of means using sample t-test. The data were considered significant at $\mathrm{p}=0.05$.

The human and dog population, including free-roaming dogs with undetermined ownership status was estimated for the study area, using a capture-recapture approach and the Lincoln-Petersen estimator inorder to determine total vaccination coverage in the dog population (Chao et al., 2001; Efford et al., 2012).

The estimation is based on the number of marked (capture) and unmarked populations counted separately during the recapturing through household survey and transect study. For the estimation of the dog population, number of dogs recorded at the vaccination centres $\left(\mathrm{n}_{1}\right)$, total number of dogs recorded $\left(\mathrm{n}_{2}\right)$ and total number of dogs found marked in the recapturing phase $(\mathrm{m})$ were considered. For the estimation of the human population number of family members (number of members in the families to which the dogs belong) recorded at the vaccination centres $\left(\mathrm{n}_{1}\right)$, total number of family members $\left(\mathrm{n}_{2}\right)$ and number of family members (the total number of people in the dog-owning households that attended the vaccination centre) of marked dogs $(\mathrm{m})$ in the recapturing phase were noted. The total population size $(\mathrm{N})$ is calculated as: $\mathrm{N}=\sum \underline{\mathrm{n}}_{1} \underline{\mathrm{n}}_{2} / \sum \mathrm{m}($ Chao et al., 2001).

Ethical clearance for the study was obtained from the Ethics Review Committee of the Faculty of Medicine, University of Colombo.

\section{RESULTS}

\section{Populations captured and recaptured}

A total of 2508 dogs presented to temporary vaccination centres during the period of survey was marked with red collars after vaccination. Total number of family members (number of members in the families in which the dogs belong) recorded at that time was 8690 . From the 2933, owned, free-roaming dogs and freeroaming dogs with undetermined ownership counted through the transect line count, 1413 had been marked at the temporary vaccination centre.

There were 2207 households in the four DS divisions and all were included in the study during the recapture phase. There were $838 \mathrm{dogs}$ and the mean age of the sample dog population was 3.5 years.

\section{Management of dogs by the owners}

During the household survey, it was found that 552 $(66 \%)$ dogs were kept restrained by caging 245 (29\%) or tying 307 (37\%). Remaining 286 (34\%) owned dogs were allowed to roam freely during the day. Among 552 confined dogs, 425 (77\%) were vaccinated and out of 286 unconfined owned dogs, only 154 (53.8\%) were vaccinated (Table 1). The percentage of females $(34.3 \%)$ were higher than that of males $(29.1 \%)$ in free-roaming dogs (dogs who are allowed to roam freely out of the household premises without restrictions).

\section{Dog population estimations and Dog anti-rabies vaccination coverage}

Owned dog (confined and free- roaming) population was estimated as 3630 (95\% CI: 3465-3793). The tabulated vaccination coverage of these dogs was $69 \%$ $(n=2508)$. Out of the total confined dogs, $77 \%$ were vaccinated whereas it is only $54 \%$ in the dogs allowed for free-roaming. The dog population including owned, free- 
Dog ecology and dog anti-rabies vaccination

Table 1: Household information obtained from respondents in households in study areas

\begin{tabular}{lccc}
\hline Variable & Frequency & Percentage $(\%)$ & ARV coverage \\
\hline $\begin{array}{l}\text { Households with dogs } \\
\text { Sex }\end{array}$ & 0.375 & 37.5 & \\
$\quad$ Male & 662 & 78.9 & $69 \%$ \\
Female & 176 & 21.1 & $66 \%$ \\
Age & & & $65 \%$ \\
$\quad$ 1 year & 132 & 15.7 & $65 \%$ \\
$\quad$ 1 year & 706 & 84.2 & $68 \%$ \\
Breed & & & $78 \%$ \\
Local & 759 & 90.5 & $83 \%$ \\
Other & 79 & 9.4 & $71 \%$ \\
Rearing method & & & $53 \%$ \\
Caged & 245 & 29.2 & 36.6 \\
Leashed & 307 & 34.1 & \\
Free & 286 & & \\
\hline
\end{tabular}

Number of dogs 838; Number of respondents 2207

roaming dogs and free-roaming dogs with undetermined ownership, was estimated as 5205 (95\% CI: 5000-5401) in the study area and calculated vaccination coverage was $48 \%(2508 / 5205)$. Only $58.8 \%(n=17)$ of the owned dogs under 3 months of age had been vaccinated.

The vaccination coverage was low in local breeds $(68 \%, n=517)$ compared to purebred dogs $(78 \%, n=62)$. From the domestic dogs included in the survey, 69\% $(n=579)$ were vaccinated previously through the National anti-rabies control program and $5.25 \%(n=42)$ were vaccinated by the private sector. Further, survey findings revealed that $34 \%$ of the owned dogs were allowed to roam freely during the day and the vaccination coverage of this population of dogs were significantly lower than that of confined dogs, either leashed or caged $(\mathrm{P}<0.005)$.

\section{Human population}

Number of family members recorded at the vaccination centres were 8690 . The household survey of the study included 2207 households, which includes 8650 family members. Out of the 2207 households 774 houses had dogs with 3382 family members. The estimated human population of the area surveyed was 34,901 (95\% CI: $33,624.56-36,177.85)$. The average number of people in a family was 4 per household, however, it ranged from 1 to 14.

The ratio of the total dog population to total human was 1:6.7 and owned dog population to human ratio was 1:9.6.

\section{DISCUSSION}

This survey was conducted after 7 years of a similar study conducted in the country to study dog population ecology and vaccination coverage of dogs against rabies. We were able to identify several important features of dog ecology and vaccination coverage that have a direct relevance for the designing of appropriate and effective dog rabies control strategies in Sri Lanka.

The study shows that vaccination coverage among the total dog population as $48 \%$. Among less than three months old owned dogs $58.8 \%$ were vaccinated. The vaccination coverage was found low $(68 \%)$ in local breeds compared to purebred dogs $78 \%$.

For estimating dog population densities in relation to human population, WHO recommendation is to compile 500 to 5000 households in a major survey (Lwanga et al.,1991; WHO/WSPA,1990). Since this study includes 2207 households, sample size of the study is sufficient to obtain an accurate estimation.

Another important aspect noted was vaccination coverage of local breeds was comparatively lower than that of other breeds (pure breed or cross breeds). This may be attributed to the low priority given to this breed by most of the owners.

Mean age of the sample dog population was found to be 3.5 years, and in general, the majority of dogs in the developing world is very young and short-lived (De Balogh et al., 1933; Davlin et al., 2012). A lack of responsible pet ownership towards caring for dogs and lack of facilities could be the possible reasons for this observation. This could lead to changes in the dog turnover rate, thus affecting the total vaccination coverage. Vaccination coverage should exceed $70 \%$ as per WHO recommendation to acquire protective levels of herd immunity among the dog population in order to eliminate dog rabies in an endemic country (WHO, 2013). Our total vaccination coverage $(48 \%)$ is below the recommended level. Under normal circumstances, vaccination programs are conducted only for a half a day on working days (Monday to Friday) in a particular place by the National anti-rabies control program. The principal reason for vaccination coverage of $69 \%$ is partly because of the difficulty in bringing $34 \%$ of the owned free 
roaming dogs to the vaccination centres within the short time period. There were instances where certain household members, ie.elders or females, could not catch/ restrain owned free-roaming dogs due to the dogs' temperament. Also there is a group of dog owners who do not present their dogs for vaccination due to negligence. These limitation could have overcome by increasing the duration of vaccination centres, employing dog catchers, and by educating owners about the methods of restraining dogs.

A study conducted in Bali, Indonesia has reported that at least $40 \%$ of vaccination coverage should be maintained in an island for 4 to 5 years to eliminate rabies effectively (Townsend et al., 2013). Therefore, the present vaccination coverage may be sufficient to eliminate rabies if we can maintain it sustainable among the total dog population for an appropriate period.

According to the results obtained in the household survey, a higher percentage of the owned dogs were males $(79 \%)$, which was similar $(65.7 \%$ domestic dogs were males) to what was observed previously in the Kalamulla Public Health Inspectorate (PHI) area in Kalutara District, Western Province, Sri Lanka in 1996 (Pimburage et al., 2011). An interviewer based survey conducted in 2002 in Thungson District of Thailand found $65 \%$ owned dogs were males (Kongkaew et al., 2004). Keeping male dogs in houses compared to female dogs could be a reason for this. This is to avoid the nuisance of owning a female dog in oestrus or to get rid of unwanted puppies.

Higher percentage of vaccination coverage achieved among confined $(77 \%)$ dogs in the survey is similar to vaccination coverage $(88 \%-70 \%)$ achieved in three different zones in urban N'Djaména,Chad (Kayali et al., 2006). This may be due to the majority of owning dog population is relatively easily accessible for free vaccination campaigns(Townsend et al., 2013). In the study it was found that $34 \%$ of the owned dogs are allowed to roam freely. In contrast to this, a previous study conducted in 1997 in the Mirigama DS division of Gampaha district, Western Province (WP), Sri Lanka, found $39.6 \%$ of dogs were either chained or caged and $60.4 \%$ were free-roaming (Matter et al., 2000). This variation may have resulted from the increased animal birth control programs conducted since 2008 by the Ministry of Health which provided better chances to control unwanted breeding which reduces the dog population. However, presence of unvaccinated, freeroaming owned dogs and free-roaming dogs with undetermined ownership is a potential challenge for sustaining the herd immunity and poses a higher risk of spreading rabies among dogs, to other domestic animals and to human.

\section{CONCLUSION}

Insufficient vaccination coverage and failure to maintain a herd immunity could be considered as the major drawbacks for the elimination of rabies from the country.Therefore, innovative planning for providing a second opportunity to get the dogs vaccinated free of charge is recomended to the campaign. Vaccination coverage can be further improved if there are vaccine centres conducting in weekends and public holidays. In order to improve vaccination coverage an incentive such as a colourful dog collar would be a way to attract dog owners to vaccination points. During annual mass vaccination campaigns, there should be a method to include dogs of all three categories i.e. owned confined, owned free-roaming and dogs with undetermined ownership status and daily monitoring of vaccination coverage is recommended.

Considering the confinement status practiced, allowing dogs to roam freely outside the household premises should be discouraged. This could potentially be acquired through implementing educational programs and new legislations bound by legal means. Animal birth control program should also strengthen to reach elimination of unwanted breeding of dogs in the community.

\section{ACKNOWLEDGEMENTS}

We gratefully acknowledge the financial support provided by the World Health Organization of Sri Lanka for this study.

\section{REFERENCES}

Awoyomi, O.J., Adeyemi, F.S., Awoyomi, F.S. (2007). Socioeconomic factors associated with nonvaccination of dogs against rabies in Ibadan, Nigeria. Nig Vet J , 28:5963

Beran, W.B. (1982). Ecology of dogs in the Central Philippines in relation to rabies control efforts. Comp Immunol Microbiol Infect Dis 5:265-270. https://doi.org/10.1016/0147-9571(82)90047-9

Bogel, K., Joshi, D.D. (1990). Accessibility of dog populations for rabies control in Kathmandu, Nepal. Bulletin of the World Health Organization 68: 611617

Briggs, D., Hanlon, C.A. (2007). World Rabies Day: focusing attention on a neglected disease. Veterinary Record 161:288-289.

https://doi.org/10.1136/vr.161.9.288

Chao, A., Tsay,P.K., Lin,S.H., Shau, W.Y., Chao, D.Y.( 2001). The applications of capture-recapture models to epidemiological data, Statistics in Medicine, volume 20: 31233157. https://doi.org/10.1002/sim.996

Coleman, P. G. and Dye, C. (1996). Immunization coverage required to prevent outbreaks of dog rabies. Vaccine, 14(3), 185186.https://doi.org/10.1016/0264410X(95)00197-9

Davlin, S.L., Vonville, H.M. (2012). Canine rabies vaccination and domestic dog population characteristics in the developing world: a systematic revi ew. Vac cine, 30 (24):3492-502. https://doi.org/10.1016/j.vaccine.2012.03.069

DeBalogh, K.M., Wandeler, A.I., Meslin, F.X. (1993). A dog ecology study in an urban and a semi-rural area of 
Zambia. Onderstepoort J Vet Res 60:437443.

Department of census and statistics Sri Lanka. http://www.statistics.gov.lk

Efford, M.G.,Fewster, R.M. (2013). Estimating population size by spatially explicit capture-recapture. Oikos 122: 918-928. doi: 10.1111/j.16000706.2012.20440.

Gbeminiyi,R.O., Jarlath, U., Umoh, Asabe, A.D (2014). Demographic and Ecological Survey of Dog Population in Aba, Abia State, Nigeria. ISRN Veterinary Science. Https://doi.org/10.1155/2014/806849

Hampson,K., Laurent,C., Tiziana, L., Maganga, S., Alexia,K., Michaël, A., Jacques, B., Jesse, D., Blanton (2015). Estimating the Global Burden of Endemic Canine Rabies.PLOS Negl Trop https://doi.org/10.1371/journal.pntd.0003709

Kaare, M., Lembo, T.,Hampson, K., Ernest, E., Estes, A., Mentzel, C., Cleaveland, S (2009) Rabies control in rural Africa: evaluating strategies for effective domestic dog vaccination. Vaccine 27:152-160. https://doi.org/10.1016/j.vaccine.2008.09.054

Kayali, U., Mindekem, R., Hutton, G., Ndoutamia, A. G.,Zinsstag, J. (2006). Coverage of pilot parenteral vaccination campaign against canine rabies in N'Djaména, Chad, Bulletin of the World Health Organization 2003;81:739-744

Knobel, D.L., Laurenson, M.K., Kazwala, R.R., Boden, L.A., Cleaveland, S. (2008) A cross-sectional study of factors associated with dog ownership in $\mathrm{T}$ a $\mathrm{n} \mathrm{z}$ a $\mathrm{ni}$ a. B M C Ve t $\mathrm{R}$ e s $4: 5$ https://doi.org/10.1186/1746-6148-4-5

Kongkaew, W., Coleman, P., Pfeiffer, D. U.,Antarasena, C., Thiptara, A. (2004) Vaccination coverage and epidemiological parameters of the owned-dog population in Thungsong district Thailand Prev Vet Med 65: 105-115. https://doi.org/10.1016/j.prevetmed.2004.05.009

Lwanga, S., K.,Lemeshow, S. (1991) Sample size determination in health studies. A practical manual world health organization, Geneva.

Malerczyk,C. (2012) Rabies Pre-Exposure Vaccination in Rabies Endemic Countries. J Vaccines Vaccin 3:e114 https://doi.org/10.4172/2157-7560.1000e114

Matter, H.C.,Wandeler, A.I.,Neuenschwander, B.E.,Harischandra, P.A.L.,Meslin, F. X. (2000) Study of the dog population and the rabies control activities in the Mirigama area of Sri Lanka. ActaTropica 75:95108.https://doi.org/10.1016/s0001-706x(99)00085-6

Nanayakkara, S., Smith, J.S., Rupprecht, C. E. (2003) Rabies in Sri Lanka: splendid isolation. Emerg Infect Dis 9:36871. https://doi.org/10.3201/eid0903.020545

Oboegbulemand, S.I.,Nwakonobi, I. E. (1989) Population density and ecology of dogs in Nigeria: a pilot study RevueScientifique et Technique de Office
International des Epizooties, vol. 8, pp 733745.https://doi.org/10.20506/rst.8.3.426

Pimburage, R. M. S., Balasuriya, A., Amunugama,R.S.K.,Gunatilake, M., Edirisuriye, D.T. (2011) An audit on dog anti-rabies immunization and sterilization program conducted in a National Institute of Health Sciences field practice area, $3^{\text {rd }}$ Rabies In Asia Conference: RIACON;Poster presentation.

Prevention and Control of Rabies in the South-East Asia Region (July 2004) World Health Organization, Regional Office for South East Asia. New Delhi

Robinson, L.E., Miranda, M.E., Miranda, N.L., Childs, J.E. (1996) Evaluation of a canine rabies vaccination campaign and characterization of owned-dog populations in the Philippines. Southeast Asian J Trop Med Public Health. 27:250256.

Rupprecht, C.E., Barrett, J., Briggs, D., Cliquet, F., Fooks, A. R., Lumlertdacha, B., Meslin, F.X.,Muler, T., Nel, L.H., Schneider, C., Tordo, N., Wandeler, A. I. (2008). Can rabies be eradicated? DevBiol (Basel) 131:95122.

Tenzin, Wangdi, K., Ward, M.P. (2012) Human and animal rabies prevention and control cost in Bhutan, 2001-2008: The cost-benefit of dog rabies elimination. Vaccine 31: 260-270.

https://doi.org/10.1016/j.vaccine.2012.05.023

Tepsumethanon, W., Polsuwan, C., Lumlertdaecha, B., Khawplod,P., Hemachudha, T., Chutivongse, S., Wilde, H., Chiewbamrungkiat, M., Phanuphak, P. (1991). Immune response to rabies in Thai dogs: a preliminary r e p o r t. Va c c i n e 9: 62 $7-630$. https://doi.org/10.1016/0264-410X(91)90186-A

Touihri, L., Zaouia, I., Elhili, K., Dellagi, K., Bahloul, C . (2011). Evaluation of mass vaccination campaign coverage against rabies in dogs in Tunisia. Zoonoses $\mathrm{Public} \mathrm{Health} 58(2): 110-118$. Https://doi.org/10.1111/j.1863-2378.2009.01306.x

Townsend, S.E., Sumantra, I.P., Pudjiatmoko,Bagus, G.N.,Brum, E.,Cleaveland, S. (2013). Designing Programs for Eliminating Canine Rabies from Islands: Bali, Indonesia as a Case Study. PLOS Negl Trop Dis 7(8): e2372. oi:10.1371/journal.pntd.0002372Vol. 14 Issue 2, p122.

WHO/WSPA, Guidelines for Dog Population Management. World Health Organization/World Society for the Protection of Animals, WHO/ZOON/90.165, World Health Organization, Geneva, Switzerland, 1990.

Wilde, H., Khawplod, P., Khamoltham, T., Hemachudha, T., Tepsumethanon., V., Lumlerdacha, B., Mitmoonpitak, C., Sitprija, V. ( 2005). Rabies control in South and Southeast Asia. Vaccine 23: 2284228 .

World Health Organization (2013). World Health 